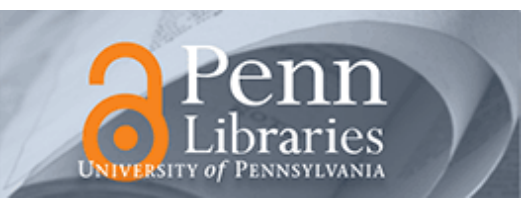

University of Pennsylvania

ScholarlyCommons

March 1988

\title{
Radiation Characteristics of a Source in a Thin Substrate Mounted Over a Dielectric Medium
}

\author{
Nader Engheta \\ University of Pennsylvania, engheta@ee.upenn.edu \\ Charles Elachi \\ California Institute of Technology
}

Follow this and additional works at: https://repository.upenn.edu/ese_papers

\section{Recommended Citation}

Nader Engheta and Charles Elachi, "Radiation Characteristics of a Source in a Thin Substrate Mounted Over a Dielectric Medium", . March 1988.

Copyright 1988 IEEE. Reprinted from IEEE Transactions on Antennas and Propagation, Volume 36, Issue 3, March 1988, pages 322-330.

This material is posted here with permission of the IEEE. Such permission of the IEEE does not in any way imply IEEE endorsement of any of the University of Pennsylvania's products or services. Internal or personal use of this material is permitted. However, permission to reprint/republish this material for advertising or promotional purposes or for creating new collective works for resale or redistribution must be obtained from the IEEE by writing to pubs-permissions@ieee.org. By choosing to view this document, you agree to all provisions of the copyright laws protecting it.

This paper is posted at ScholarlyCommons. https://repository.upenn.edu/ese_papers/180

For more information, please contact repository@pobox.upenn.edu. 


\title{
Radiation Characteristics of a Source in a Thin Substrate Mounted Over a Dielectric Medium
}

\begin{abstract}
The radiation pattern of a line source is calculated for the case where the source is lying on the top or the bottom surface of a lossless dielectric substrate that is mounted on the top of a semi-infinite dielectric medium. It is found that in both cases the pattern along interfaces has a null; that the pattern in the upper semi-infinite medium has a single lobe; and that the pattern in the lower semi-infinite medium has many lobes, the number of which varies with the substrate thickness. It is shown that in both cases the power radiated into the lower medium is more than that radiated into the upper medium. Applications of this calculation in remote sensing, microstrip antenna technology, and antenna arrays are discussed.

\section{Comments}

Copyright 1988 IEEE. Reprinted from IEEE Transactions on Antennas and Propagation, Volume 36, Issue 3, March 1988, pages 322-330.

This material is posted here with permission of the IEEE. Such permission of the IEEE does not in any way imply IEEE endorsement of any of the University of Pennsylvania's products or services. Internal or personal use of this material is permitted. However, permission to reprint/republish this material for advertising or promotional purposes or for creating new collective works for resale or redistribution must be obtained from the IEEE by writing to pubs-permissions@ieee.org. By choosing to view this document, you agree to all provisions of the copyright laws protecting it.
\end{abstract}




\title{
Radiation Characteristics of a Source in a Thin Substrate Mounted Over a Dielectric Medium
}

\author{
NADER ENGHETA, MEMBER, IEEE, AND CHARLES ELACHI, SENIOR MEMBER, IEEE
}

\begin{abstract}
The radiation pattern of a line source is calculated for the case where the source is lying on the top or the bottom surface of a lossless dielectric substrate that is mounted on the top of a semi-infinite dielectric medium. It is found that in both cases the pattern along interfaces has a null; that the pattern in the upper semi-infinite medium has a single lobe; and that the pattern in the lower semi-infinite medium has many lobes, the number of which varies with the substrate thickness. It is shown that in both cases the power radiated into the lower medium is more than that radiated into the upper medium. Applications of this calculation in remote sensing, microstrip antenna technology, and antenna arrays are discussed.
\end{abstract}

\section{INTRODUCTION}

A MONG the problems of remote sensing, the interface problems are the ones that mostly attract the attention of physicists and engineers who work in electrodynamics. One of these problems is the problem of reflection and refraction of the electromagnetic wave illuminating a rough ground surface covered with a dielectric layer. The top surface of the layer can also be rough. There are different theoretical models that can simplify the geometry of the problem and thus can lead to finding the reflection and refraction of the wave. One of the techniques that can be useful in solving the problems of the scattering from rough surfaces is the equivalent current source technique that was used by Marcuse [1] and also by Elachi and Yeh [2]. In using this technique, the slightly rough surface is replaced by a smooth surface and the equivalent sources located on the interface of the smooth surface. We call these sources the interfacial sources. Therefore, the original problem reduces to the problem of finding the radiation of sources that lie on interfaces. In our previous paper [3], we found the radiation patterns and emitted power of a line source lying along the interface of two lossless dielectric half-spaces. In the present paper, we will obtain the radiation patterns of a line source lying along the top or the bottom surface of a dielectric layer over a smooth surface.

In microstrip antenna technology, it is often necessary to calculate the radiation characteristic of dipoles printed on a dielectric substrate. The substrate is usually mounted on a conducting ground plane. However, the ground plane can, in some special cases, be replaced with a high permittivity

Manuscript received July 9, 1986; revised December 24, 1987

N. Engheta was with the California Institute of Technology, Pasadena, CA $\mathrm{He}$ is now with the Moore School of Electrical Engineering, University of Pennsylvania, Philadelphia, PA 19104-6390.

C. Elachi is with the Jet Propulsion Laboratory, California Institute of Technology, Pasadena, CA 91109.

IEEE Log Number 8718766. substrate (such as in aperture-coupled microstrip antennas with two parallel substrates).

Also, it will be shown that there is a potential application in generating grating lobes similar to that of antenna arrays using a single dipole sandwiched between two dielectric regions. The present paper will discuss the radiation properties of radiating sources in these cases.

Fig. 1 illustrates the geometry of the problem. The substrate is a lossless homogeneous dielectric slab whose dielectric constant $\epsilon_{1}$ is greater than that of free space, i.e., $\epsilon_{1}>\epsilon_{0}$. The lower half-space is also a lossless homogeneous dielectric with permittivity $\epsilon_{2}$ greater than $\epsilon_{1}$. In Section II, we will consider the line source located along the bottom surface of the layer. We will find the radiation pattern of the line source in this configuration. In Section III, we will examine the problem for the case of the line source lying along the top surface of the layer. Section IV will contain the interpretation of the results in terms of ray optics. Finally, in Section V, a brief summary and applications of the problem will be given.

\section{The Line Source is on the Bottom Surface of the SubSTRATE}

\section{A. Formulation of the Problem}

To formulate the problem mathematically, we introduce a Cartesian coordinate system $x, y, z$ wherein the $z$ axis lies along the axis of the line source, and the plane of the interface of the layer, whose index of refraction is $n_{1}=\sqrt{\epsilon_{1} / \epsilon_{0}}$ and the lower medium, whose index of refraction is $n_{2}=\sqrt{\epsilon_{2} / \epsilon_{0}}$, is given by the coordinate surface $y=0$. The plane interface of the layer and the upper medium is denoted by a plane $y=D$, where $D$ is the thickness of the layer. Moreover, to handle the far-zone field, which must have the form of a cylindrical wave, we also introduce a cylindrical coordinate system $\rho, \phi$, $z$, where $\rho \cos \phi=x, \rho \sin \phi=y$ and $-\pi \leqslant \phi \leqslant \pi$ (Fig. 2).

We use the MKS system of units and assume that the current density of the line source is given by

$$
\mathbf{J}=\mathbf{e}_{z} I \delta(x) \delta(y) e^{-i \omega t}
$$

where $\mathbf{e}_{z}$ is the unit vector along the $z$ axis, $\delta(x)$ and $\delta(y)$ are Dirac delta functions, and $I$ is the total current. We take the index of refraction to be 1 in the upper medium $(y>D)$; and $n_{1}>1$ in the substrate with thickness $D(0 \leqslant y \leqslant D)$; and $n_{2}$ $>n_{1}$ in the lower half-space $(y<0)$. Although this means that the upper medium is a vacuum, and the substrate and the lower medium are dielectric, our analysis will be true for any three dielectric regions whose indices of refraction are such 


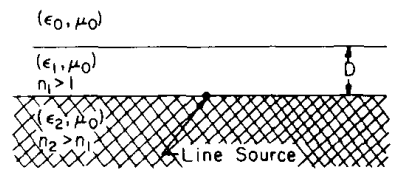

(a)

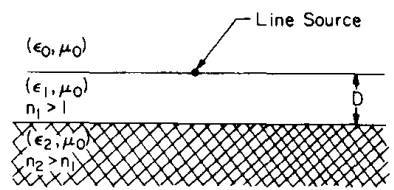

(b)

Fig. 1. (a) Line source is located on bottom surface of lossless dielectric slab. (b) Line source is located on top surface of slab.

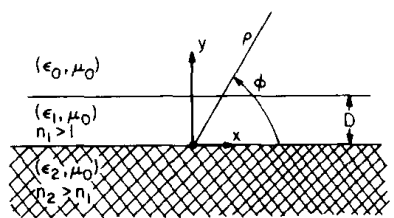

Fig. 2. Line source lies along $z$ axis. In the upper half-space $(y>D)$ the index of refraction $n$ is equal to 1 , in slab $(0<y<D) n_{1}$ is greater than 1 , and in lower half-space $(y<0) n_{2}$ is greater than $n_{1}$.

that $n_{2}>n_{1}>n_{0}$. The results will also be valid if the indices of refraction are in the ratio of $n_{2}$ to $n_{1}$ to 1 .

From the symmetry of the configuration, it is evident that all the electromagnetic field components are independent of $z$, and consequently there are only three components $E_{z}(\rho, \phi)$, $H_{\rho}(\rho, \phi)$, and $H_{\phi}(\rho, \phi)$. From the Maxwell equations, we can write

$$
\begin{gathered}
H_{\rho}=\frac{1}{i \omega \mu} \frac{1}{\rho} \frac{\partial}{\partial \phi} E_{z} \\
H_{\phi}=\frac{i}{\omega \mu} \frac{\partial}{\partial \rho} E_{z} .
\end{gathered}
$$

Therefore, since $E_{z}$ is the only component of the electric field and since the magnetic field components can be derived from $E_{z}$, our problem can be formulated in terms of $E_{z}$ alone.

From Maxwell's field equations $\nabla \times \mathbf{E}=i \omega \mu \mathbf{H}$ and $\nabla \times$ $\mathbf{H}=\mathbf{J}-i \omega \epsilon \mathbf{E}$, it follows that

$$
\nabla \times \nabla \times \mathbf{E}-k^{2} \mathbf{E}=i \omega \mu \mathbf{J},
$$

where $k^{2}=\omega^{2} \mu \epsilon$. We also know that $\nabla \cdot(\epsilon \mathbf{E})=0$. Using vector identities, we get $\nabla \epsilon \cdot \mathbf{E}+\epsilon \nabla \cdot \mathbf{E}=0$. Because here $\nabla \epsilon$ is perpendicular to $\mathbf{E}$, the term $\nabla \epsilon \cdot \mathbf{E}$ disappears, and we have

$$
\nabla \cdot \mathbf{E}=0 .
$$

From (1), (4), and (5), $E_{z}$ must satisfy

$$
\nabla^{2} E_{z}+k^{2} E_{z}=-i \omega \mu I \delta(x) \delta(y)
$$

where $\mu=\mu_{0}$ everywhere,

$$
k^{2}=\omega^{2} \mu_{0} \epsilon_{0}=k_{0}^{2} \text { for } y>D,
$$

$$
k^{2}=\omega^{2} \mu_{0} \epsilon_{1}=n_{1}^{2} k_{0}^{2} \text { for } 0 \leqslant y \leqslant D,
$$

and

$$
k^{2}=\omega^{2} \mu_{0} \epsilon_{2}=n_{2}^{2} k_{0}^{2} \text { for } y<0 .
$$

$\mu_{0}$ and $\epsilon_{0}$ denote the permeability and the dielectric constant of free space. We denote $\mathbf{E}$ and $\mathbf{H}$ in the upper medium by $\mathbf{E}_{0}$ and $\mathbf{H}_{0}$, in the substrate by $\mathbf{E}_{1}$ and $\mathbf{H}_{1}$ and in the lower semi-infinite medium by $\mathbf{E}_{2}$ and $\mathbf{H}_{2}$, respectively.

Therefore, we must find the solution of (6) which gives outwardly moving cylindrical wave in the far zone and satisfies the boundary conditions $E_{z 0}=E_{z 1}$ and $H_{x 0}=H_{x 1}$ along the plane $y=D$, and $E_{z 1}=E_{z 2}$ and $H_{x 1}=H_{x 2}$ along the plane $y=0$.

\section{B. Solutions of the Equations}

To solve (6), we write $E_{z}$ as a Fourier integral, i.e.,

$$
E_{z}(x, y)=\int_{-\infty}^{\infty} V(y, h) e^{i h x} d h .
$$

By substituting (7) into (6) and by recalling that

$$
\delta(x)=\frac{1}{2 \pi} \int_{-\infty}^{\infty} e^{i h x} d h,
$$

we get the differential equation for $V(y, h)$, i.e.,

$$
\frac{d^{2}}{d y^{2}} V+\left(k^{2}-h^{2}\right) V=\frac{-i \omega \mu_{0} I \delta(y)}{2 \pi}
$$

where for $y<0$ we have $k^{2}=n_{2}^{2} k_{0}^{2}$ and denote $V$ by $V_{2}$, and for $0 \leqslant y \leqslant D$ we have $k^{2}=n_{1}^{2} k_{0}^{2}$ and denote $V$ by $V_{1}$; and

$$
\frac{d^{2}}{d y^{2}} V+\left(k_{0}^{2}-h^{2}\right) V=0, \text { for } y>D
$$

where we denote $V$ by $V_{0}$ for $y>D$.

The solution to (9) and (10) must also satisfy the radiation condition. Therefore, the appropriate solutions of (9) and (10) have the form

$$
V_{0}(y, h)=a_{0} \exp \left(-\sqrt{h^{2}-k_{0}^{2}} y\right)
$$

$$
V_{1}(y, h)=a_{1} \exp \left(-\sqrt{h^{2}-n_{1}^{2} k_{0}^{2}} y\right)+b_{1} \exp \left(\sqrt{h^{2}-n_{1}^{2} k_{0}^{2}} y\right)
$$

$$
V_{2}(y, h)=a_{2} \exp \left(\sqrt{h^{2}-\overline{n_{2}^{2}} k_{0}^{2}} y\right) .
$$

To determine $a_{0}, a_{1}, b_{1}$, and $a_{2}$ we use the boundary conditions for $E_{z}$. Accordingly, we must have

$$
\begin{aligned}
& V_{0}=V_{1} \text { at } y=D \\
& V_{1}=V_{2} \text { at } y=0 .
\end{aligned}
$$

To find the other relations among $a_{0}, a_{1}, b_{1}$, and $a_{2}$, we integrate (9) with respect to $y$ from $-\Delta y$ to $+\Delta y$, and also 
integrate (10) with respect to $y$ from $D-\Delta y$ to $D+\Delta y . \Delta y$ is vanishingly small quantity and $V$ is a continuous function of $y$ throughout the range of integration. Therefore, we get

$$
\begin{gathered}
\left.\frac{d V}{d y}\right|_{y=\Delta y}-\left.\frac{d V}{d y}\right|_{y=-\Delta y}=\frac{-i \omega \mu_{0}}{2 \pi} I \\
\left.\frac{d V}{d y}\right|_{y=D+\Delta y}=\left.\frac{d V}{d y}\right|_{y=D-\Delta y} .
\end{gathered}
$$

Considering the relations (11a)-(11c), (12a), (12b), and (13a), (13b), we obtain $a_{0}, a_{2}$. From (7), (11a), (11c) and a knowledge of $a_{0}$ and $a_{2}$, we find

$$
\begin{aligned}
E_{z 0}= & \int_{-\infty}^{\infty} \frac{i \omega \mu_{0} I}{\pi}\left(\sqrt{h^{2}-n_{1}^{2} k_{0}^{2}}\right) \\
& \cdot\left\{\sqrt { h ^ { 2 } - k _ { 0 } ^ { 2 } } \left[\sqrt { h ^ { 2 } - n _ { 1 } ^ { 2 } k _ { 0 } ^ { 2 } } \left(\exp \left(\sqrt{h^{2}-n_{1}^{2} k_{0}^{2}} D\right)\right.\right.\right. \\
& \left.+\exp \left(-\sqrt{h^{2}-n_{1}^{2} k_{0}^{2}} D\right)\right)-\sqrt{h^{2}-n_{2}^{2} k_{0}^{2}} \\
& \left.\cdot\left(\exp \left(-\sqrt{h^{2}-n_{1}^{2} k_{0}^{2}} D\right)-\exp \left(\sqrt{h^{2}-n_{1}^{2} k_{0}^{2}} D\right)\right)\right] \\
& +\sqrt{h^{2}-n_{1}^{2} k_{0}^{2}}\left[\sqrt { h ^ { 2 } - n _ { 1 } ^ { 2 } k _ { 0 } ^ { 2 } } \left(\exp \left(\sqrt{h^{2}-n_{1}^{2} k_{0}^{2}} D\right)\right.\right. \\
& \left.-\exp \left(-\sqrt{h^{2}-n_{1}^{2} k_{0}^{2}} D\right)\right)+\sqrt{h^{2}-n_{2}^{2} k_{0}^{2}} \\
& \left.\left.\cdot\left(\exp \left(\sqrt{h^{2}-n_{1}^{2} k_{0}^{2}} D\right)+\exp \left(-\sqrt{h^{2}-n_{1}^{2} k_{0}^{2}} D\right)\right)\right]\right\}^{-1} \\
& \cdot \exp \left(\sqrt{h^{2}-k_{0}^{2}} D+i h x-\sqrt{h^{2}-k_{0}^{2}} y\right) d h
\end{aligned}
$$

and

$$
\begin{aligned}
E_{z 2}= & \int_{-\infty}^{\infty} \frac{i \omega \mu_{0} I}{2 \pi}\left[\sqrt { h ^ { 2 } - n _ { 1 } ^ { 2 } k _ { 0 } ^ { 2 } } \left(\exp \left(\sqrt{h^{2}-n_{1}^{2} k_{0}^{2}} D\right)\right.\right. \\
& \left.+\exp \left(-\sqrt{h^{2}-n_{1}^{2} k_{0}^{2}} D\right)\right)+\sqrt{h^{2}-k_{0}^{2}} \\
& \left.\cdot\left(\exp \left(\sqrt{h^{2}-n_{1}^{2} k_{0}^{2}} D\right)-\exp \left(-\sqrt{h^{2}-n_{1}^{2} k_{0}^{2}} D\right)\right)\right] \\
& \cdot\left\{\sqrt { h ^ { 2 } - k _ { 0 } ^ { 2 } } \left[\sqrt { h ^ { 2 } - n _ { 1 } ^ { 2 } k _ { 0 } ^ { 2 } } \cdot \left(\exp \left(\sqrt{h^{2}-n_{1}^{2} k_{0}^{2}} D\right)\right.\right.\right. \\
& \left.+\exp \left(-\sqrt{h^{2}-n_{1}^{2} k_{0}^{2}} D\right)\right)-\sqrt{h^{2}-n_{2}^{2} k_{0}^{2}} \\
& \left.\cdot\left(\exp \left(-\sqrt{h^{2}-n_{1}^{2} k_{0}^{2}} D\right)-\exp \left(\sqrt{h^{2}-n_{1}^{2} k_{0}^{2}} D\right)\right)\right] \\
& +\sqrt{h^{2}-n_{1}^{2} k_{0}^{2}}\left[\sqrt { h ^ { 2 } - n _ { 1 } ^ { 2 } k _ { 0 } ^ { 2 } } \left(\exp \left(\sqrt{h^{2}-n_{1}^{2} k_{0}^{2}} D\right)\right.\right. \\
& \left.-\exp \left(-\sqrt{h^{2}-n_{1}^{2} k_{0}^{2}} D\right)\right)+\sqrt{h^{2}-n_{2}^{2} k_{0}^{2}} \\
& \left.\left.\cdot\left(\exp \left(\sqrt{h^{2}-n_{1}^{2} k_{0}^{2}} D\right)+\exp \left(-\sqrt{h^{2}-n_{1}^{2} k_{0}^{2}} D\right)\right)\right]\right\}^{-1} \\
& \cdot \exp \left(i h x+\sqrt{h^{2}-n_{2}^{2} k_{0}^{2}} y\right) d h .
\end{aligned}
$$

To find the radiation patterns in the upper medium $(y>D)$ and the lower half-space $(y \leqslant 0)$, we resort to an asymptotic evaluation of the integrals.

\section{Radiation Patterns}

To determine the radiation patterns, it is convenient to use the cylindrical coordinates $(\rho, \phi)$. Accordingly, in the integrals of (14) and (15), we replace $x, y$ by $\rho \cos \phi$ and $\rho \sin$ $\phi$, respectively. Thus we obtain

$$
\begin{aligned}
E_{z 0}(\rho, \phi)= & \int_{-\infty}^{\infty} A_{0}(h) \\
& \cdot \exp \left(i h \rho \cos \phi-\sqrt{h^{2}-k_{0}^{2}} \rho \sin \phi\right) d h \\
E_{z 2}(\rho, \phi)= & \int_{-\infty}^{\infty} A_{2}(h) \\
& \cdot \exp \left(i h \rho \cos \phi+\sqrt{h^{2}-n_{2}^{2} k_{0}^{2}} \rho \sin \phi\right) d h
\end{aligned}
$$

where $A_{0}(h)$ and $A_{2}(h)$ are shorthands for parts of integrands in (14) and (15).

In the integral representation of $E_{z 0}(\rho, \phi)$, we divide the range of integration into three subranges, i.e.,

$$
E_{z 0}=\int_{-\infty}^{-k_{0}} f(h) d h+\int_{-k_{0}}^{+k_{0}} f(h) d h+\int_{k_{0}}^{\infty} f(h) d h
$$

where $f(h)$ is a shorthand for the integrand. For $k_{0} \rho \rightarrow \infty$, it can be seen that the integrals for the subranges $-\infty<h<$ $-k_{0}$ and $k_{0}<h<+\infty$ are negligibly small compared to the integral for the middle subrange $\left(-k_{0}<h<+k_{0}\right)$ [4]. Therefore, by introducing the variable $\alpha$, which is defined by $\sin \alpha=h / k_{0}$, we obtain

$E_{z 0}=\int_{-\pi / 2}^{\pi / 2} \tilde{A_{0}}(\alpha) \exp \left[i k_{0} \rho \sin (\alpha+\phi)\right] d \alpha$,

for $k_{0} \rho \rightarrow \infty$

and $0<\phi<\pi$, where $\tilde{A}_{0}(\alpha)$ is found by substituting $h=k_{0}$ $\sin \alpha$ in function $A_{0}(h)$. We apply the method of stationary phase, and we get the following expressions for $E_{z 0}$ in the far zone of the upper medium $(y>D)$ :

$$
\begin{aligned}
E_{z 0}= & \frac{\omega \mu_{0} I}{\sqrt{2 \pi k_{0} \rho}} \sin \phi \sqrt{n_{1}^{2}-\cos ^{2} \phi} \\
& \cdot\left\{\operatorname { s i n } \phi \left[i \sqrt{n_{2}^{2}-\cos ^{2} \phi} \sin \left(k_{0} D \sqrt{n_{1}^{2}-\cos ^{2} \phi}\right)\right.\right. \\
& \left.-\sqrt{n_{1}^{2}-\cos ^{2} \phi} \cos \left(k_{0} D \sqrt{n_{1}^{2}-\cos ^{2} \phi}\right)\right] \\
& -\sqrt{n_{1}^{2}-\cos ^{2} \phi}\left[\sqrt{n_{2}^{2}-\cos ^{2} \phi} \cos \left(k_{0} D \sqrt{n_{1}^{2}-\cos ^{2} \phi}\right)\right. \\
& \left.\left.-i \sqrt{n_{1}^{2}-\cos ^{2} \phi} \sin \left(k_{0} D \sqrt{n_{1}^{2}-\cos ^{2} \phi}\right)\right]\right\}^{-1} \\
& \cdot \exp \left[i k_{0}(\rho-D \sin \phi)-i \pi / 4\right]
\end{aligned}
$$

for $k_{0} \rho \rightarrow \infty$, and $0<\phi<\pi$.

Similarly, we divide the range of integration of the integral representation of $E_{z 2}(\rho, \phi)$ into subranges. Thus we write

$E_{z 2}(\rho, \phi)$

$$
\begin{aligned}
= & \int_{-\infty}^{-n_{2} k_{0}} U(h) d h+\int_{-n_{2} k_{0}}^{-n_{1} k_{0}} U(h) d h \\
& +\int_{-n_{1} k_{0}}^{-k_{0}} U(h) d h+\int_{-k_{0}}^{k_{0}} U(h) d h+\int_{k_{0}}^{n_{1} k_{0}} U(h) d h \\
& +\int_{n_{1} k_{0}}^{n_{2} k_{0}} U(h) d h+\int_{n_{2} k_{0}}^{\infty} U(h) d h
\end{aligned}
$$


where $U(h)$ is a shorthand for the integrand. The first and seventh integrals are negligibly small for all values of $\phi$ in the lower half-space $(-\pi<\phi<0)[4]$. Defining the variable $\alpha$ by $\sin \alpha=h /\left(n_{2} k_{0}\right)$, we can invoke the method of stationary phase. To have a leading term of order $1 / \sqrt{n_{2} k_{0} \rho}$ for $n_{2} k_{0} \rho \rightarrow \infty$, the stationary point must be in the interval of integration. Therefore, if $\phi$ lies in the intervals $-\pi \leqslant \phi \leqslant$ $-\pi+\phi_{c 1}$ and $-\phi_{c 1} \leqslant \phi \leqslant 0$, where $\cos \phi_{c 1}=n_{1} / n_{2}$, the contribution to the far-zone field will come from the second and sixth integrals, respectively. However, if $\phi$ lies in the intervals $-\pi+\phi_{c 1} \leqslant \phi \leqslant-\pi+\phi_{c 2}$ and $-\phi_{c 2} \leqslant \phi \leqslant$ $-\phi_{c 1}$, where $\cos \phi_{c 2}=1 / n_{2}$, the leading term for the far-zone field will come from the third and fifth integrals, respectively. Finally, if $\phi$ lies in the sector $-\pi+\phi_{c 2} \leqslant \phi \leqslant-\phi_{c 2}$, the leading term will result from the fourth integral. Therefore, for the dihedral region $\left(n_{2} k_{0} \rho \rightarrow \infty\right.$, and $-\pi+\phi_{c 2} \leqslant \phi \leqslant$ $-\phi_{c 2}$, we have

$$
\begin{aligned}
E_{z 2}= & \frac{\omega \mu_{0} I}{\sqrt{2 \pi n_{2} k_{0} \rho}} n_{2} \sin \phi\left[\sqrt{n_{1}^{2}-n_{2}^{2} \cos ^{2} \phi}\right. \\
& \cdot \cos \left(k_{0} D \sqrt{n_{1}^{2}-n_{2}^{2} \cos ^{2} \phi}\right)-i \sqrt{1-n_{2}^{2} \cos ^{2} \phi} \\
& \left.\cdot \sin \left(k_{0} D \sqrt{n_{1}^{2}-n_{2}^{2} \cos ^{2} \phi}\right)\right]\left\{\sqrt{1-n_{2}^{2} \cos ^{2} \phi}\right. \\
& \cdot\left[\sqrt{n_{1}^{2}-n_{2}^{2} \cos ^{2} \phi} \cos \left(k_{0} D \sqrt{n_{1}^{2}-n_{2}^{2} \cos ^{2} \phi}\right)\right. \\
& \left.+i n_{2} \sin \phi \sin \left(k_{0} D \sqrt{n_{1}^{2}-n_{2}^{2} \cos ^{2} \phi}\right)\right] \\
& -\sqrt{n_{1}^{2}-n_{2}^{2} \cos ^{2} \phi}\left[i \sqrt{n_{1}^{2}-n_{2}^{2} \cos ^{2} \phi}\right. \\
& \cdot \sin \left(k_{0} D \sqrt{n_{1}^{2}-n_{2}^{2} \cos ^{2} \phi}\right)+n_{2} \sin \phi \\
& \left.\left.\cdot \cos \left(k_{0} D \sqrt{n_{1}^{2}-n_{2}^{2} \cos ^{2} \phi}\right)\right]\right\}^{-1} \\
& \cdot \exp \left(i n_{2} k_{0} \rho-i \pi / 4\right) .
\end{aligned}
$$

For the two sectors $-\pi+\phi_{c 1} \leqslant \phi \leqslant-\pi+\phi_{c 2}$ and $-\phi_{c 2}$ $\leqslant \phi \leqslant-\phi_{c 1}$, we get

$$
\begin{aligned}
E_{z 2}= & \frac{-\omega \mu_{0} I}{\sqrt{2 \pi n_{2} k_{0} \rho}} n_{2} \sin \phi\left[\sqrt{n_{1}^{2}-n_{2}^{2} \cos ^{2} \phi}\right. \\
& \cdot \cos \left(k_{0} D \sqrt{n_{1}^{2}-n_{2}^{2} \cos ^{2} \phi}\right)+\sqrt{n_{2}^{2} \cos ^{2} \phi-1} \\
& \left.\cdot \sin \left(k_{0} D \sqrt{n_{1}^{2}-n_{2}^{2} \cos ^{2} \phi}\right)\right]\left\{\sqrt{n_{1}^{2}-n_{2}^{2} \cos ^{2} \phi}\right. \\
& \cdot\left[i \sqrt{n_{1}^{2}-n_{2}^{2} \cos ^{2} \phi} \sin \left(k_{0} D \sqrt{n_{1}^{2}-n_{2}^{2} \cos ^{2} \phi}\right)\right. \\
& \left.+n_{2} \sin \phi \cos \left(k_{0} D \sqrt{n_{1}^{2}-n_{2}^{2} \cos ^{2} \phi}\right)\right] \\
& -i \sqrt{n_{2}^{2} \cos ^{2} \phi-1}\left[i n_{2} \sin \phi\right. \\
& \cdot \sin \left(k_{0} D \sqrt{n_{1}^{2}-n_{2}^{2} \cos ^{2} \phi}\right)+\sqrt{n_{1}^{2}-n_{2}^{2} \cos ^{2} \phi} \\
& \left.\left.\cdot \cos \left(k_{0} D \sqrt{n_{1}^{2}-n_{2}^{2} \cos ^{2} \phi}\right)\right]\right\}-1 \\
& \cdot \exp \left(i n_{2} k_{0} \rho-i \pi / 4\right)
\end{aligned}
$$

and for the other two sectors $\left(-\pi \leqslant \phi \leqslant-\pi+\phi_{c 1}\right.$ and

$$
\begin{aligned}
-\phi_{c 1} \leqslant & \phi \leqslant 0), \text { we obtain } \\
E_{z 2}= & \frac{-i \omega \mu_{0} I}{\sqrt{2 \pi n_{2} k_{0} \rho}} n_{2} \sin \phi\left[\sqrt{n_{2}^{2} \cos ^{2} \phi-n_{1}^{2}}\right. \\
& \cdot \cosh \left(k_{0} D \sqrt{n_{2}^{2} \cos ^{2} \phi-n_{1}^{2}}\right)+\sqrt{n_{2}^{2} \cos ^{2} \phi-1} \\
& \left.\cdot \sinh \left(k_{0} D \sqrt{n_{2}^{2} \cos ^{2} \phi-n_{1}^{2}}\right)\right]\left\{\sqrt{n_{2}^{2} \cos ^{2} \phi-1}\right. \\
& \cdot\left[\sqrt{n_{2}^{2} \cos ^{2} \phi-n_{1}^{2}} \cosh \left(k_{0} D \sqrt{n_{2}^{2} \cos ^{2} \phi-n_{1}^{2}}\right)\right. \\
& \left.+i n_{2} \sin \phi \sinh \left(k_{0} D \sqrt{n_{2}^{2} \cos ^{2} \phi-n_{1}^{2}}\right)\right] \\
& +\sqrt{n_{2}^{2} \cos ^{2} \phi-n_{1}^{2}}\left[\sqrt{n_{2}^{2} \cos ^{2} \phi-n_{1}^{2}}\right. \\
& \cdot \sinh \left(k_{0} D \sqrt{n_{2}^{2} \cos ^{2} \phi-n_{1}^{2}}+i n_{2} \sin \phi\right. \\
& \left.\left.\cdot \cosh \left(k_{0} D \sqrt{n_{2}^{2} \cos ^{2} \phi-n_{1}^{2}}\right)\right]\right\}-1 \\
& \cdot \exp \left(i n_{2} k_{0} \rho-i \pi / 4\right) .
\end{aligned}
$$

In the far-zone region, the magnetic and electric fields have the following relations:

$$
H_{\phi 0}=-\sqrt{\frac{\epsilon_{0}}{\mu_{0}}} E_{z 0}
$$

in the upper medium $(y>D)$; and

$$
H_{\phi 2}=-n_{2} \sqrt{\frac{\epsilon_{0}}{\mu_{0}}} E_{z 2}
$$

in the lower semi-infinite medium $(y<0)$.

By recalling that the time-averaged Poynting vector $\mathbf{S}=$ $1 / 2 \operatorname{Re}\left(\mathbf{E} \times \mathbf{H}^{*}\right)$, one can get

$$
S_{\rho 0}=\frac{1}{2} \sqrt{\frac{\epsilon_{0}}{\mu_{0}}}\left|E_{z 0}\right|^{2}
$$

in the upper medium, and

$$
S_{\rho_{2}}=\frac{1}{2} n_{2} \sqrt{\frac{\epsilon_{0}}{\mu_{0}}}\left|E_{z 2}\right|^{2}
$$

in the lower half-space. Equations (20) and (22)-(24) present $E_{z 0}$ and $E_{z 2}$.

The radiation patterns of this source are plotted using (27) and (28) (see Figs. 3-6 for radiation patterns plotted for different values of $D / \lambda_{0}$ ). We see that at the interface the radiation patterns disappear, and that in the upper medium, the radiation pattern consists of a single lobe whose maximum lies along the line $\phi=\pi / 2$ and has the value

$$
\begin{aligned}
\left(S_{\rho 0}\right)_{\max }=\frac{\omega \mu_{0} I^{2}}{4 \pi \rho} n_{1}^{2}\left[n_{1}^{2}(1\right. & \left.+n_{2}\right)^{2} \cos ^{2}\left(n_{1} k_{0} D\right) \\
& \left.+\left(n_{2}+n_{1}^{2}\right)^{2} \sin ^{2}\left(n_{1} k_{0} D\right)\right]^{-1} .
\end{aligned}
$$

The value of the Poynting vector in the lower semi-infinite 


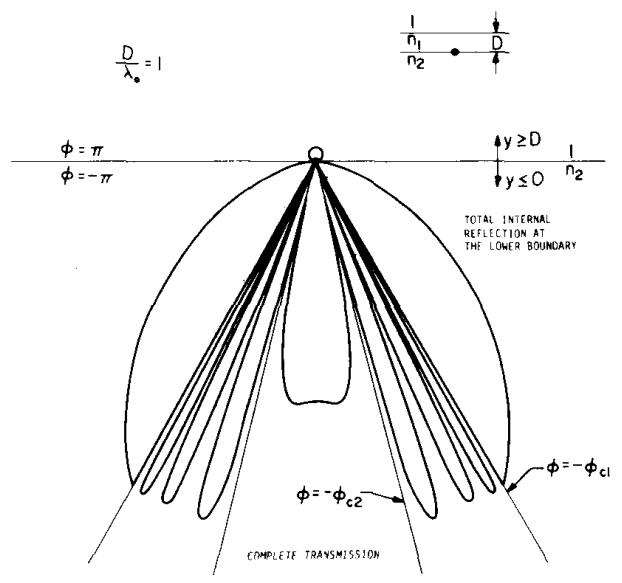

Fig. 3. Radiation patterns (linear scale) for the case where the line source is located on bottom surface of the slab. Here, $n_{1}=2, n_{2}=4, D / \lambda_{0}=1, \cos$ $\phi_{c 1}=1 / 2$, and $\cos \phi_{c 2}=1 / 4$.

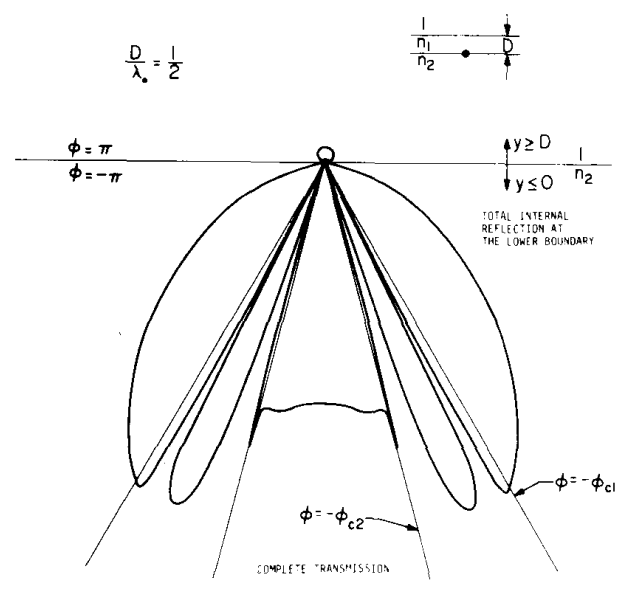

Fig. 4. Radiation patterns (linear scale) for the case where the line source is located on bottom surface of the slab. Here, $n_{1}=2, n_{2}=4, D / \lambda_{0}=1 / 2$, $\cos \phi_{c 1}=1 / 2$, and $\cos \phi_{c^{\prime} 2}=1 / 4$.

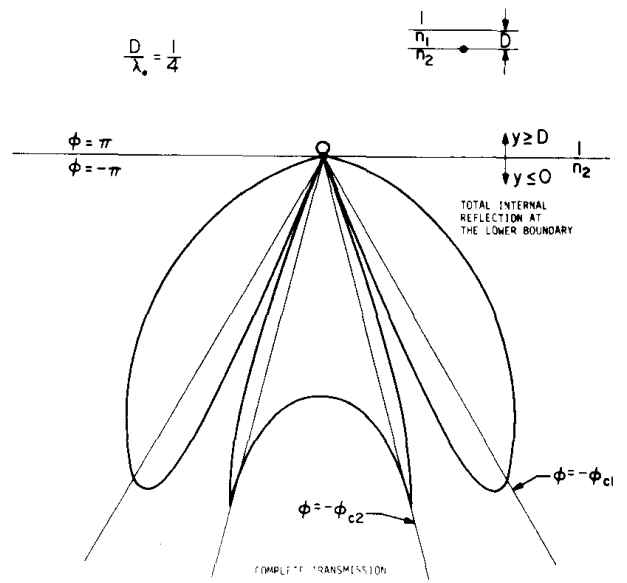

Fig. 5. Radiation patterns (linear scale) for the case where the line source is located on bottom surface of the slab. Here, $n_{1}=2, n_{2}=4, D / \lambda_{0}=1 / 4$, $\cos \phi_{c 1}=1 / 2$, and $\cos \phi_{c 2}=1 / 4$.

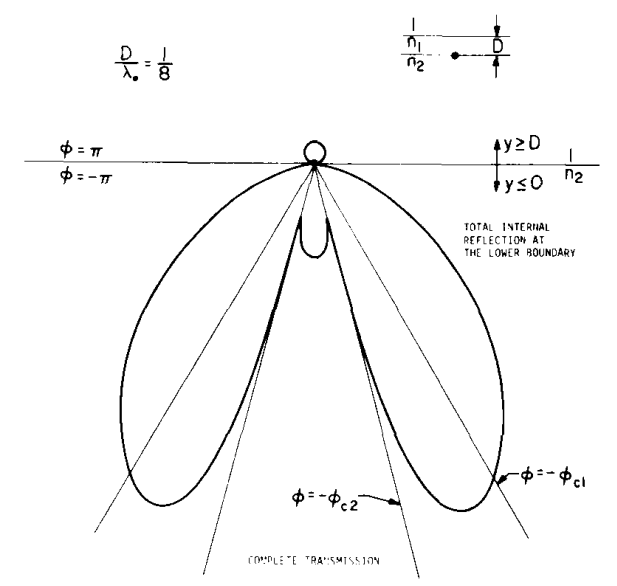

Fig. 6. Radiation patterns (linear scale) for the case where the line source is located on bottom surface of the slab. Here, $n_{1}=2, n_{2}=4, D / \lambda_{0}=1 / 8$, $\cos \phi_{\mathrm{c} 1}=1 / 2$, and $\cos \phi_{\mathrm{c} 2}=1 / 4$.

medium at $\phi=-\pi / 2$ is

$$
\begin{aligned}
\left(S_{\rho 2}\right)_{\phi=-\pi / 2}= & \frac{\omega \mu_{0} I^{2}}{4 \pi \rho} n_{2}^{2}\left[n_{1}^{2} \cos ^{2}\left(n_{1} k_{0} D\right)\right. \\
& \left.+\sin ^{2}\left(n_{1} k_{0} D\right)\right] /\left\{n_{1}^{2}\left(n_{2}+1\right)^{2}\right. \\
& \cdot \cos ^{2}\left(n_{1} k_{0} D\right)+\left(n_{1}^{2}+n_{2}\right)^{2} \\
& \left.\cdot \sin ^{2}\left(n_{1} k_{0} D\right)\right\} .
\end{aligned}
$$

Now, we can examine the limiting cases. When the substrate on top of the source disappears, $D$ equals zero. Therefore, one can have the following expressions for (29) and (30) for $D \rightarrow$ 0 , i.e.,

$$
\begin{gathered}
\left(S_{\rho 0}\right)_{\max } \rightarrow \frac{\omega \mu_{0} I^{2}}{4 \pi \rho} \frac{1}{\left(1+n_{2}\right)^{2}} \text { for } D \rightarrow 0 \\
\left(S_{\rho 2}\right)_{\phi=-\pi / 2} \rightarrow \frac{\omega \mu_{0} I^{2}}{4 \pi \rho} \frac{n_{2}^{2}}{\left(1+n_{2}\right)^{2}} \text { for } D \rightarrow 0 .
\end{gathered}
$$

These are exactly the time-averaged Poynting vectors that would be radiated by the line source if it lay between two semiinfinite dielectric half-spaces. This was shown in [3]. Another limiting case is where $n_{1}=n_{2}=1$. One gets the following relations for (29) and (30) when $n_{1}=n_{2} \rightarrow 1$

$$
\begin{aligned}
& \left(S_{\rho 0}\right)_{\max } \rightarrow \frac{\omega \mu_{0} I^{2}}{16 \pi \rho} \quad \text { for } n_{1}=n_{2} \rightarrow 1 \\
& \left(S_{\rho 2}\right)_{\phi=-\pi / 2} \rightarrow \frac{\omega \mu_{0} I^{2}}{16 \pi \rho}
\end{aligned}
$$

which is the time-averaged Poynting vector that would be radiated by the line source if it were in free space. As was shown, the radiation patterns of such a source approach that of the limiting cases already studied.

We see that the number of lobes in the radiation pattern in the lower half-space varies with the thickness $D$. As $D$ increases, the number of lobes being in the sectors $-\pi+\phi_{\mathrm{cl}}$ $<\phi<-\pi+\phi_{c 2}$ and $-\phi_{c 2}<\phi<-\phi_{c 1}$ increases. This is due to the multiple reflection inside the slab. The effect is 
similar to the interference effect. When $D$ increases, the difference between path lengths of rays reflected inside the slab varies more rapidly with the angle. Therefore, a small change in the angle can cause a significant difference among path lengths inside the slab, resulting in a significant change in the radiation pattern. This effect is well observed in the sectors $-\pi+\phi_{c 1}<\phi<-\pi+\phi_{c 2}$ and $-\phi_{c 2}<\phi<-\phi_{c 1}$ and is not significant in other angular regions. This is because in the downward central part the difference among path lengths is not so sensitive to angular variation. In the other angular regions, the total internal reflections would also affect this behavior. The ray optical description explained in Section IV will be useful in interpreting some of these results.

The radiation in the bottom central lobe can have a drastic change as $D$ varies from $\lambda_{0} / 4$ to $\lambda_{0} / 8$ (compare Figs. 5 and 6 ). This can be explained as follows: in Fig. $5, D / \lambda_{0}=1 / 4$, and $n_{1}=2$ which means $D / \lambda_{1}=n_{1} D / \lambda_{0}=1 / 2$. However, in Fig. $6, D / \lambda_{0}=1 / 8$ which leads to $D / \lambda_{1}=1 / 4$. For Fig. 5, a round trip path of a ray in the substrate is equivalent to one wavelength in the substrate, and thus adds constructively to the other rays. However, in Fig. 6 a round-trip path is half of the wavelength in the substrate, and therefore adds destructively. That is why one gets a drastic change in the bottom central lobe changing $D$ from $\lambda_{0} / 4$ to $\lambda_{0} / 8$. The plots of radiation pattern reveal the fact that the power radiated into the lower half space is more than that radiated into the upper medium. The larger the value of $n_{2}$ is, the less power is radiated into the upper medium for the same value of $I$ [3], [5]. Therefore,

$$
\left(S_{\rho 0}\right)_{\max } \rightarrow 0 \quad \text { for } n_{2} \rightarrow \infty .
$$

III. The Line Source is on the Top Surface of the SUBSTRATE

Using the same Cartesian and cylindrical coordinate systems and applying a similar approach for the problem shown in Fig. 1(b), we get the expressions of $S_{\rho 0}$ and $S_{\rho 2}$ in the far zone, i.e.,

$$
\begin{aligned}
S_{\rho 0}= & \frac{\omega \mu_{0} I^{2}}{4 \pi \rho} \sin ^{2} \phi\left[\left(n_{1}^{2}-\cos ^{2} \phi\right) \cos ^{2}\left(k_{0} D \sqrt{n_{1}^{2}-\cos ^{2} \phi}\right)\right. \\
& \left.+\left(n_{2}^{2}-\cos ^{2} \phi\right) \sin ^{2}\left(k_{0} D \sqrt{n_{1}^{2}-\cos ^{2} \phi}\right)\right] \\
& \cdot\left[\left(n_{1}^{2}-\cos ^{2} \phi\right) \cos ^{2}\left(k_{0} D \sqrt{n_{1}^{2}-\cos ^{2} \phi}\right)\right. \\
& \cdot\left(\sqrt{n_{2}^{2}-\cos ^{2} \phi}+\sin \phi\right)^{2}+\sin ^{2}\left(k_{0} D \sqrt{n_{1}^{2}-\cos ^{2} \phi}\right) \\
& \left.\cdot\left(\sqrt{n_{2}^{2}-\cos ^{2} \phi} \sin \phi+n_{1}^{2}-\cos ^{2} \phi\right)^{2}\right]^{-1}
\end{aligned}
$$

for $k_{0} \rho \rightarrow \infty$ and $0<\phi<\pi$;

$$
\begin{aligned}
S_{\rho 2}= & \frac{\omega \mu_{0} I^{2}}{4 \pi \rho} n_{2}^{2} \sin ^{2} \phi\left(n_{1}^{2}-n_{2}^{2} \cos ^{2} \phi\right) \\
& \cdot\left[\left(n_{1}^{2}-n_{2}^{2} \cos ^{2} \phi\right) \cos ^{2}\left(k_{0} D \sqrt{n_{1}^{2}-n_{2}^{2} \cos ^{2} \phi}\right)\right. \\
& \cdot\left(\sqrt{1-n_{2}^{2} \cos ^{2} \phi}-n_{2} \sin \phi\right)^{2} \\
& +\sin ^{2}\left(k_{0} D \sqrt{n_{1}^{2}-n_{2}^{2} \cos ^{2} \phi}\right) \\
& \left.\cdot\left(n_{2} \sqrt{1-n_{2}^{2} \cos ^{2} \phi} \sin \phi-n_{1}^{2}+n_{2}^{2} \cos ^{2} \phi\right)^{2}\right]^{-1}
\end{aligned}
$$

$$
\text { for } \begin{aligned}
n_{2} k_{0} \rho \rightarrow \infty \text { and }-\pi+\phi_{c 2} \leqslant \phi \leqslant-\phi_{c 2} \\
\qquad \begin{aligned}
S_{\rho 2}= & \frac{\omega \mu_{0} I^{2}}{4 \pi \rho} n_{2}^{2} \sin ^{2} \phi\left(n_{1}^{2}-n_{2}^{2} \cos ^{2} \phi\right) \\
& \cdot\left\{n _ { 2 } ^ { 2 } \operatorname { s i n } ^ { 2 } \phi \left[\sqrt{n_{1}^{2}-n_{2}^{2} \cos ^{2} \phi}\right.\right. \\
& \cdot \cos \left(k_{0} D \sqrt{n_{1}^{2}-n_{2}^{2} \cos ^{2} \phi}\right)+\sqrt{n_{2}^{2} \cos ^{2} \phi-1} \\
& \left.\cdot \sin \left(k_{0} D \sqrt{n_{1}^{2}-n_{2}^{2} \cos ^{2} \phi}\right)\right]^{2} \\
& +\left(n_{1}^{2}-n_{2}^{2} \cos ^{2} \phi\right)\left[\sqrt{n_{2}^{2} \cos ^{2} \phi-1}\right. \\
& \cdot \cos \left(k_{0} D \sqrt{n_{1}^{2}-n_{2}^{2} \cos ^{2} \phi}\right)-\sqrt{n_{1}^{2}-n_{2}^{2} \cos ^{2} \phi} \\
& \left.\left.\cdot \sin \left(k_{0} D \sqrt{n_{1}^{2}-n_{2}^{2} \cos ^{2} \phi}\right)\right]^{2}\right\}^{-1}
\end{aligned}
\end{aligned}
$$

for $n_{2} k_{0} \rho \rightarrow \infty$, and $-\pi+\phi_{c 1} \leqslant \phi \leqslant-\pi+\phi_{c 2}$ and $-\phi_{c 2}$ $\leqslant \phi \leqslant-\phi_{c 1} ;$ and

$$
\begin{aligned}
S_{\rho 2}= & \frac{\omega \mu_{0} I^{2}}{4 \pi \rho} n_{2}^{2} \sin ^{2} \phi\left(n_{2}^{2} \cos ^{2} \phi-n_{1}^{2}\right) \\
& \cdot\left\{( n _ { 2 } ^ { 2 } \operatorname { c o s } ^ { 2 } \phi - n _ { 1 } ^ { 2 } ) \left[\sqrt{n_{2}^{2} \cos ^{2} \phi-n_{1}^{2}}\right.\right. \\
& \cdot \sinh \left(k_{0} D \sqrt{n_{2}^{2} \cos ^{2} \phi-n_{1}^{2}}\right)+\sqrt{n_{2}^{2} \cos ^{2} \phi-1} \\
& \left.\cdot \cosh \left(k_{0} D \sqrt{n_{2}^{2} \cos ^{2} \phi-n_{1}^{2}}\right)\right]^{2} \\
& +n_{2}^{2} \sin ^{2} \phi\left[\sqrt{n_{2}^{2} \cos ^{2} \phi-n_{1}^{2}}\right. \\
& \cdot \cosh \left(k_{0} D \sqrt{n_{2}^{2} \cos ^{2} \phi-n_{1}^{2}}+\sqrt{n_{2}^{2} \cos ^{2} \phi-1}\right. \\
& \left.\left.\cdot \sinh \left(k_{0} D \sqrt{n_{2}^{2} \cos ^{2} \phi-n_{1}^{2}}\right)\right]^{2}\right\}^{-1}
\end{aligned}
$$

for $n_{2} k_{0} \rho \rightarrow \infty,-\pi \leqslant \phi \leqslant-\pi+\phi_{c 1}$ and $-\phi_{c\}} \leqslant \phi \leqslant 0$.

From (36)-(39), we can sketch the radiation patterns of the line source in this configuration (see Figs. $7-10$ for radiation patterns plotted for different values of $D / \lambda_{0}$ ). We see that the radiation pattern at the interface disappears. We also notice that in the upper medium the radiation pattern is a lobe whose maximum does not always lie along the line $\phi=\pi / 2$. In the lower medium, it can be seen that the number of lobes also varies with the thickness $D$. As $D$ increases, the number of lobes increases. The power radiated into the lower half-space is greater than that radiated into the upper medium. In fact, $S_{\rho 0}$ $\rightarrow 0$ as $n_{2} \rightarrow \infty$.

For this configuration, we can also examine the limiting cases. As the substrate thickness approaches zero, i.e., $D \rightarrow$ 0 , one can obtain the following expressions for (36),

$$
S_{\rho 0}=\frac{\omega \mu_{0} I^{2}}{4 \pi \rho} \frac{\left(\sin ^{2} \phi-\sin \phi \sqrt{n_{2}^{2}-\cos ^{2} \phi}\right)^{2}}{\left(n_{2}^{2}-1\right)^{2}}
$$

which is the time-averaged power flux density that would be radiated by the line source if it lay between two semi-infinite dielectric half spaces. Similarly, one can obtain the corresponding expressions for $S_{\rho 2}$ and examine the limiting cases for $S_{\rho 2}$ [3]. When $n_{2}=n_{1},(36)$ turns out to be (40), indicating a 


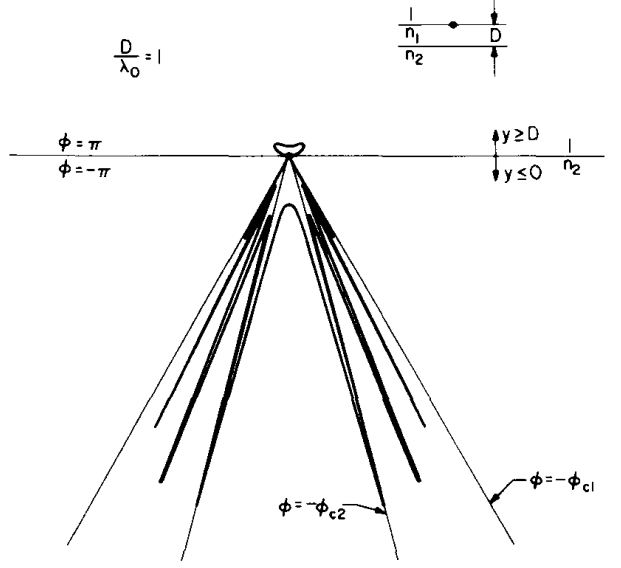

Fig. 7. Radiation patterns (linear scale) for the case where the line source is located on top surface of the slab. Here, $n_{1}=2, n_{2}=4, D / \lambda_{0}=1, \cos \phi_{c 1}$ $=1 / 2$, and $\cos \phi_{c 2}=1 / 4$.

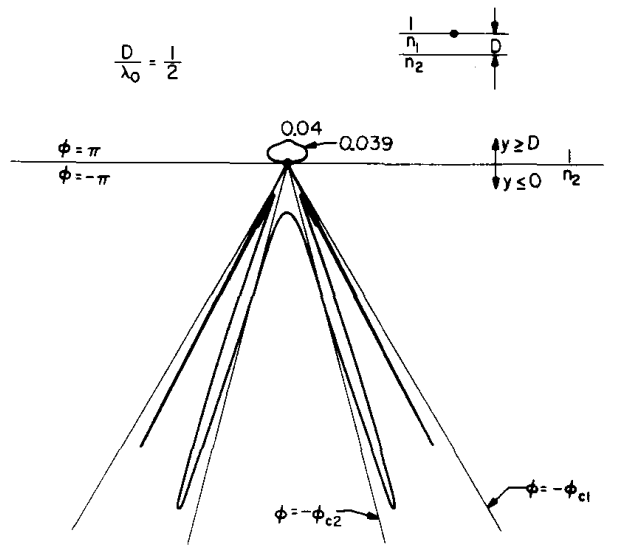

Fig. 8. Radiation patterns (linear scale) for the case where the line source is located on top surface of the slab. Here, $n_{1}=2, n_{2}=4, D / \lambda_{0}=1 / 2, \cos$ $\phi_{c 1}=1 / 2$, and $\cos \phi_{c 2}=1 / 4$.

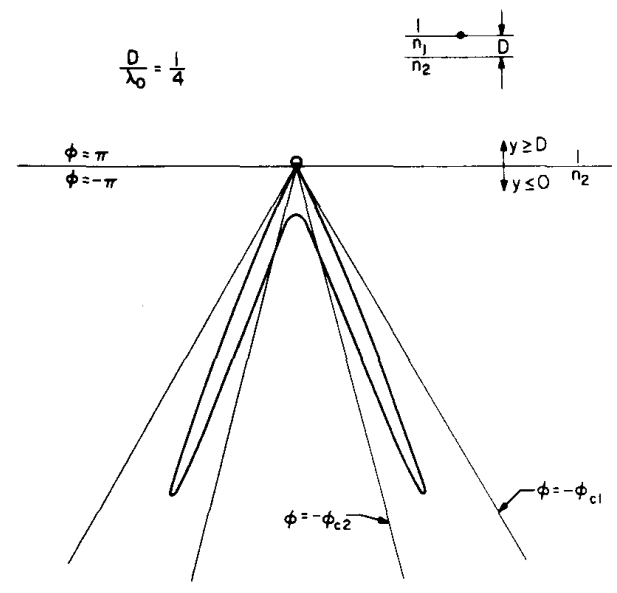

Fig. 9. Radiation patterns (linear scale) for the case where the line source is located on top surface of the slab. Here, $n_{1}=2, n_{2}=4, D / \lambda_{0}=1 / 4$, cos $\phi_{c 1}=1 / 2$, and $\cos \phi_{c 2}=1 / 4$.

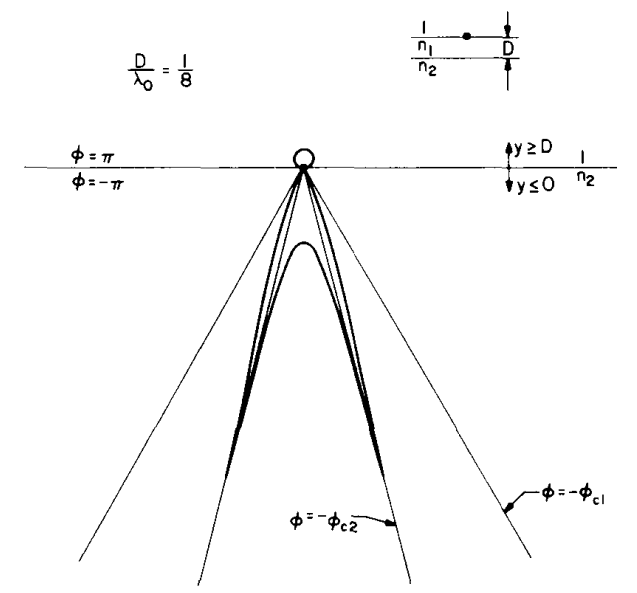

Fig. 10. Radiation patterns (linear scale) for the case where the line source is located on top surface of the slab. Here, $n_{1}=2, n_{2}=4, D / \lambda_{0}=1 / 8, \cos$ $\phi_{c 1}=1 / 2$, and $\cos \phi_{c 2}=1 / 4$.

similar behavior. When the source is on top of the substrate, the radiation in the bottom central part is mostly confined to the edges, unlike the previous case where the radiation is usually more confined to the center.

Generally, the radiation patterns in both cases reveal the fact that one can get many lobes in the lower medium by having a dielectric substrate over a medium with a different permittivity. In fact, the lobes of radiation patterns resemble the grating lobes of an interferometer, even though there is only one radiating source here. This could be a potential application of the foregoing analysis. One can use a single receiving antenna, mounted on top of a hemispherical lens with an index of refraction $n_{2}$, and put a substrate with thickness $D$ and permittivity $n_{1}<n_{2}$ on top of the antenna. The thickness $D$ can control the number of lobes. Since radiation (or receiving) pattern is much stronger in the lower medium, this can potentially be used as a receiving array. This could reduce the cost and complication of making an array using many dipoles mounted on a hemispherical lens.

\section{Ray Optical Description}

We can construct a suitable ray optical description to obtain the foregoing results. To find the results when the line source is on the bottom surface of the layer, we must take the source to be a little below the bottom surface, where the index of refraction, is $n_{2}$. The far-zone field in the upper medium ( $y>$ $D$ ) is the ray transmitted through the layer; and the far-zone field in the lower half-space is the sum of two rays: the direct ray from the source and the ray reflected by the interface (Fig. 11).

Accordingly, the far-zone field in the lower medium is given by

$$
E_{z 2}=\sqrt{\frac{P_{0}}{n_{2} \pi \rho}} \sqrt[4]{\frac{\mu_{0}}{\epsilon_{0}}}[1+R(\theta)] e^{i n_{2} k_{0} \rho}
$$

where $P_{0}$ denotes power radiated by the line in a homogeneous dielectric, $\theta$ is the angle of incidence, and $R(\theta)$ is the reflection 


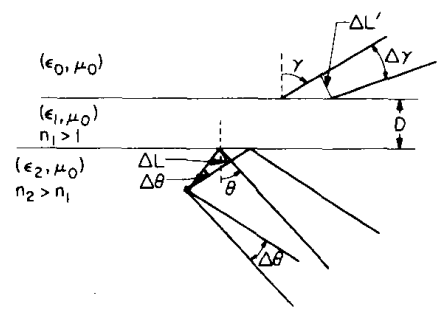

Fig. 11. Ray diagram for the case of source a little below bottom surface of slab.

coefficient for the layer, where the incident wave is in the lower medium whose index of refraction is $n_{2}$.

The far-zone field in the upper medium is given by

$$
E_{z 0}=\sqrt{\frac{P_{0}}{n_{2} \pi \rho}} \sqrt[4]{\frac{\mu_{0}}{\epsilon_{0}}} T(\gamma) \sqrt{\frac{\Delta \theta}{\Delta \gamma}} \cdot \frac{\bar{\Delta} L^{\prime}}{\Delta L} e^{i k_{0} \rho}
$$

where $\gamma$ is the angle of refraction and $T(\gamma)$ is the transmission coefficient for the layer. $\Delta \theta, \Delta \gamma, \Delta L$, and $\Delta L^{\prime}$ are illustrated in Fig. 11.

Using Snell's law and Fig. 11, we see that

$$
\frac{\Delta L^{\prime}}{\Delta L} \frac{\Delta \theta}{\Delta \gamma}=\frac{n_{2} \cos ^{2} \gamma}{n_{2}^{2}-\sin ^{2} \gamma} .
$$

Now, we write $R(\theta)$ and $T(\gamma)$ for the layer [5]; that is,

$$
\begin{aligned}
& R=\frac{r_{2}+r_{1} e^{+2 i \delta_{2}}}{1+r_{1} r_{2} e^{+2 i \delta_{2}}} \\
& T=\frac{t_{1} t_{2} e^{i \delta_{2}}}{1+r_{1} r_{2} e^{+2 i \delta_{2}}}
\end{aligned}
$$

where $r_{1}$ and $t_{1}$ are the reflection and transmission coefficients for the boundary between the layer and the upper medium when the incident wave is considered to be in the layer, $r_{2}$ and $t_{2}$ are the reflection and transmission coefficients for the boundary between the lower half-space and the layer when the incident wave is in the lower half-space, and $\delta_{2}$ is given by

$$
\delta_{2}=k_{0} D \sqrt{n_{1}^{2}-n_{2}^{2} \sin ^{2} \theta}=k_{0} D \sqrt{n_{1}^{2}-\sin ^{2} \gamma} .
$$

We can find $r_{1}, r_{2}, t_{1}$, and $t_{2}$ by using the Fresnel formulas and Snell's law. Therefore, we get

$$
\begin{gathered}
r_{1}=\frac{\sqrt{n_{1}^{2}-n_{2}^{2} \sin ^{2} \theta}-\sqrt{1-n_{2}^{2} \sin ^{2} \theta}}{\sqrt{n_{1}^{2}-n_{2}^{2} \sin ^{2} \theta}+\sqrt{1-n_{2}^{2} \sin ^{2} \theta}} \\
=\frac{\sqrt{n_{1}^{2}-\sin ^{2} \gamma}-\cos \gamma}{\sqrt{n_{1}^{2}-\sin ^{2} \gamma}+\cos \gamma} \\
r_{2}=\frac{n_{2} \cos \theta-\sqrt{n_{1}^{2}-n_{2}^{2} \sin ^{2} \theta}}{n_{2} \cos \theta+\sqrt{n_{1}^{2}-n_{2}^{2} \sin ^{2} \theta}} \\
=\frac{\sqrt{n_{2}^{2}-\sin ^{2} \gamma}-\sqrt{n_{1}^{2}-\sin ^{2} \gamma}}{\sqrt{n_{2}^{2}-\sin ^{2} \gamma}+\sqrt{n_{1}^{2}-\sin ^{2} \gamma}}
\end{gathered}
$$

$$
\begin{gathered}
t_{2}=\frac{2 \sqrt{n_{1}^{2}-\sin ^{2} \gamma}}{\sqrt{n_{1}^{2}-\sin ^{2} \gamma}+\cos \gamma} \\
t_{2}=\frac{2 \sqrt{n_{2}^{2}-\sin ^{2} \gamma}}{\sqrt{n_{2}^{2}-\sin ^{2} \gamma}+\sqrt{n_{1}^{2}-\sin ^{2} \gamma}} .
\end{gathered}
$$

In the foregoing relations, whenever we have negative values under the $\sqrt{ }$, we write $+i$ in front of the $\sqrt{ }$ and make the values positive. By substituting (46)-(50) into (44) and (45), then substituting (43)-(45) into (41) and (42), and knowing that $\phi=\pi / 2-\gamma$ and $\phi=\theta-\pi / 2$, we find $E_{z 0}$ and $E_{z 2}$. Except for the ignorable constant phase difference, using ray optical approach, we obtain $E_{z 0}$ and $E_{z 2}$ identical to the ones that we get from a field theoretic approach. We can follow a similar approach to find $E_{z 0}$ and $E_{z 2}$ for the case where the line source is on the top surface of the substrate.

Using ray optical description, one can again see the effect of slab thickness in generating numerous lobes in the lower medium due to the multiple reflection inside the slab.

\section{CONCLUSION}

Starting from the Maxwell equations, we have calculated the radiation patterns of a line source lying on the top or the bottom surface of a lossless dielectric substrate which is mounted over a dielectric medium. From our calculation, we can conclude that in both cases the radiation pattern in the upper medium has a single lobe; and that the pattern in the lower medium can have numerous lobes. The number of lobes depends on the thickness of the slab. As the thickness of the slab increases, the number of lobes in the pattern in the lower medium increases. It can be easily seen that more power is radiated into the lower half-space than into the upper halfspace.

Due to the stronger radiation and number of sharp lobes in the lower medium, this structure can potentially be used as a receiving array. Thus one can build a receiving array, using a single dipole sandwiched between a dielectric hemispherical lens with a reflection index $n_{2}$ (lower medium) and a substrate with thickness $D$ and an index of refraction $n_{1}<n_{2}$ (slab). The number of lobes can be controlled by the thickness $D$.

\section{ACKNOWLEDGMENT}

The authors would like to thank Professor Charles H. Papas of the California Institute of Technology for his helpful discussions, encouragement and support during the course of this work. They would also like to thank Ms. Lulu Penn for her careful typing of the manuscript.

\section{REFERENCES}

[1] D. Marcuse, Light Transmission Optics. New York: Van NostrandReinhold, 1972 (see also D. Marcuse, Theory of Dielectric Optical Waveguides. New York: Academic, 1974).

[2] C. Elachi and C. Yeh, "Mode conversion in periodically disturbed thin-film waveguides," J. Appl. Phys., vol. 45, pp. 3494-3499, Aug. 1974.

[3] N. Engheta, C. H. Papas, and C. Elachi, "Interface extinction and subsurface peaking of the radiation pattern of a line source," Appl. Phys., vol. B26, pp. 231-238, 1981. 
[4] C. M. Bender and S. A. Orszag, Advanced Mathematical Methods for Scientists and Engineers. New York: McGraw-Hill, 1978.

[5] R. W. P. King and G. S. Smith, Antennas in Matter. Cambridge. MA: MIT Press, 1981

[6] O. S. Heavens, Optical Properties of Thin Solid Films. New York: Dover, 1965.

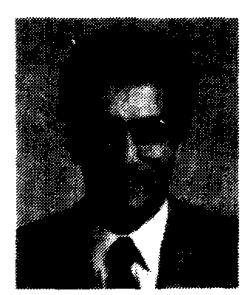

Nader Engheta (S'80-M'82) was born in Tehran, Iran, on October 8,1955 . He received the B.S. degree (with honors) in electrical engineering from the University of Tehran in 1978, the M.S. degree in electrical engineering, and the $\mathrm{Ph} . \mathrm{D}$. degrees in electrical engineering and physics from the California Institute of Technology, Pasadena, in 1979 and 1982 , respectively.

From June 1982 to June 1983, he was a Postdoctoral Research Fellow at Caltech working on the problems relevant to wave propagation, microwave and effects of wave-polarization on information gathering in remote sensing. He was also associated on a part-time basis with the Radar Remote Sensing group of Caltech's Jet Propulsion Laboratory investigating theoretical problems related to remote sensing. From June 1983 to June 1987, he was a Senior Research Scientist at Kaman Sciences Corporation, Dikewood Division, working on various problems such as high power microwave coupling to aircraft antennas, lightning effects, EMP simulator designs, effects of corona on transmission lines and extrapolation techniques in electromagnetics. Concurrently, he was a Visiting Associate at Caltech working on wave propagation in inhomogeneous and chiral media. In the spring of 1986, he was a part-time Visiting Lecturer in the Department of Electrical Engineering, University of California, Los Angeles. In July 1987, he joined the faculty of the Moore School of Electrical Engineering, University of Pennsylvania.

Dr. Engheta is a member of the American Physical Society, the American Association for the Advancement of Science, and the IEEE Antennas and Propagation, Geoscience and Remote Sensing, and Electromagnetic Compatibility Societies.

Charles Elachi (M'81-SM'82), for a photograph and biography please see page 825 of the July 1987 issue of this TRANSACTIONS. 